\title{
Embedded crack model: I. Basic formulation
}

\author{
Milan Jirásek ${ }^{*, \dagger}$ and Thomas Zimmermann \\ Laboratory of Structural and Continuum Mechanics, Department of Civil Engineering, Swiss Federal \\ Institute of Technology (EPFL), CH-1015 Lausanne, Switzerland
}

\begin{abstract}
SUMMARY
The recently emerged idea of enriching standard finite element interpolations by strain or displacement discontinuities has triggered the development of powerful techniques that allow efficient modelling of regions with highly localized strains, e.g. of fracture zones in concrete, or shear bands in metals or soils. The present paper describes a triangular element with an embedded displacement discontinuity that represents a crack. The constitutive model is formulated within the framework of damage theory, with crack closure effects and friction on the crack faces taken into account. Numerical aspects of the implementation are discussed. In a companion paper, the embedded crack approach is combined with the more traditional smeared crack approach. Copyright (c) 2001 John Wiley \& Sons, Ltd.
\end{abstract}

KEY WORDS: fracture; damage; cracking; localization; embedded discontinuity; enriched finite element

\section{INTRODUCTION}

Traditional smeared-crack models for concrete fracture suffer by stress locking, i.e. by spurious stress transfer across a widely open crack. For fixed crack models with a non-zero shear retention factor, locking is mainly due to shear stresses generated by a rotation of the principal strain axes after the crack initiation. However, locking is observed even for rotating crack models, which keep the principal axes of strain and stress aligned so that stresses tangential to the crack cannot arise. The source of this undesirable phenomenon was analysed in Reference [1]. It was shown that the spurious stress transfer is caused by a poor kinematic representation of the discontinuous displacement field around a macroscopic crack. Unless the direction of the macroscopic crack (represented by a band of cracking elements) happens to be parallel to element sides, the directions of maximum principal strain determined from the finite element interpolation at individual Gauss points deviate from the normal to the crack band. The lateral principal stress has a non-zero projection on the crack-band normal, which generates spurious cohesive forces acting across the

\footnotetext{
*Correspondence to: Milan Jirásek, Laboratory of Structural and Continuum Mechanics, Department of Civil Engineering, Swiss Federal Institute of Technology (EPFL), CH-1015 Lausanne, Switzerland

†E-mail: Milan.Jirasek@EPFL.ch
}

Contract/grant sponsor: Swiss Commission for Technology and Innovation; contract/grant number: CTI.3201.1 
macroscopic crack even at very late stages of the cracking process when the crack should be completely stress free.

Stress locking can be eliminated by improving the kinematic representation of highly localized fracture. Here we focus on techniques that insert a discontinuity (of strains or displacements) into the interior of a finite element. Such methods can be found in the literature under various names [2-11] but they share some common features. Their systematic classification and critical evaluation within a unified framework is presented in Reference [12], with the conclusion that there exist three different approaches, which can be called statically optimal symmetric (SOS), kinematically optimal symmetric (KOS), and statically and kinematically optimal non-symmetric (SKON). The SOS formulation works with a natural stress continuity condition, but it does not properly reflect the kinematics of a completely open crack. On the other hand, the KOS formulation describes the kinematic aspects satisfactorily, but it leads to an awkward relationship between the stress in the bulk of the element and the tractions across the discontinuity line. These findings justify the development of the non-symmetric SKON formulation, which combines the strong points of each of the symmetric formulations and leads to an improved numerical performance. The SKON formulation deals with a very natural stress continuity condition and is capable of properly representing complete separation at late stages of the fracturing process, without any locking effects (spurious stress transfer).

The present paper complements the theoretical investigations presented in Reference [12] by a practical implementation of a specific model with a strong (displacement) discontinuity embedded in a constant-strain triangular element. The constitutive description of a damaging interface is developed in Section 2. Section 3 gives a brief review of the SKON formulation. Numerical aspects of the implementation are discussed in Section 4. Examples of fracture simulations and extensions of the model are presented in the companion paper.

\section{CONSTITUTIVE EQUATIONS}

\subsection{Damage-based traction-separation law}

The choice of an appropriate constitutive model suitable for implementation in an element with an embedded discontinuity is affected by the type of the discontinuity. For models incorporating weak (strain) discontinuities it is sufficient to postulate a continuum stress-strain law, while models with strong discontinuities require, in addition to a stress-strain law for the bulk material, also a traction-separation law governing the behavior of the discontinuity (crack or plastic slip surface). In the present study we will focus on the latter case. It is, therefore, necessary to postulate a law that links the traction transmitted by the discontinuity to the displacement jump. As the intended area of application is concrete cracking, a natural choice is a model based on the damage theory. Such models can be conveniently derived by the thermodynamic approach, starting from an expression for the density of Helmholtz free energy. For example, for the isotropic continuum damage model with a single scalar damage parameter, the free energy density per unit volume is given by

$$
\psi(\boldsymbol{\varepsilon}, \omega)=\frac{1}{2}(1-\omega) \boldsymbol{\varepsilon}: \mathbf{D}_{e}: \boldsymbol{\varepsilon}
$$

where $\boldsymbol{\varepsilon}$ is the strain tensor, $\mathbf{D}_{e}$ is the elastic stiffness tensor, and $\omega$ is the damage parameter growing from zero (virgin material) to one (complete loss of integrity). Note that, for simplicity, we neglect the effect of temperature, tacitly assuming that the process is (at least approximately) 
isothermal. Strictly speaking, the potential (1) describes only the energy stored in the elastic deformation of the bulk material between microdefects such as cracks or voids. The complete expression for free energy should also include a term corresponding to the surface energy of the microdefects. However, the presence of this term would be important only in coupled thermomechanical problems with a substantial contribution of the mechanical dissipation to the energy balance equation. For the present purpose (a purely mechanical problem), the surface energy term can be omitted.

When constructing a traction-separation law, we have to start from an expression for free energy per unit area. The strain tensor $\boldsymbol{\varepsilon}$ is replaced by a vector e characterizing the displacement jump (separation). However, such vector describes only the inelastic part of deformation (it is identically zero before the onset of cracking), and so it is not appropriate to keep the same form of the free-energy function as for the continuum, in which the strain tensor corresponds to the sum of the elastic and inelastic deformation. Before a crack is initiated, it does not contribute to the deformation, and its 'initial' stiffness has to be considered as infinite. Consequently, the finite crack stiffness after crack initiation cannot be expressed as a scalar multiple of the initial one. Of course, it would be possible to introduce some fictitious very high initial stiffness but such an approach is not very elegant and may lead to numerical problems. If the crack trajectory is not known in advance, one would have to place such potential cracks at many different locations and with many different orientations, in order to allow the propagation of the actual crack along an arbitrary path. A much more natural description of the degradation process is obtained if one postulates the surface density of free energy,

$$
\psi(\mathbf{e}, \gamma)=\frac{1}{2 \gamma} \mathbf{e} \cdot \hat{\mathbf{D}} \cdot \mathbf{e}
$$

as a function of the separation vector, $\mathbf{e}$, and a new internal variable, $\gamma$, which is called the compliance parameter and varies from zero to infinity. In the continuum model defined by Equation (1), the compliance parameter would correspond to $\omega /(1-\omega)$. Symbol $\hat{\mathbf{D}}$ in Equation (2) denotes a second-order tensor describing the stiffness of the discontinuity (crack) at an intermediate reference state when $\gamma=1$. This state sets the scale for $\gamma$ and its choice does not affect the response of the model (in the sense that after proper rescaling of the compliance parameter the same response is obtained with any choice of the reference state).

For any possible process, the model must satisfy the dissipation inequality

$$
\mathscr{D} \equiv \mathbf{t} \cdot \dot{\mathbf{e}}-\dot{\psi} \geqslant 0
$$

where $\mathscr{D}$ is the dissipation rate (per unit area), and $\mathbf{t}$ is the traction transmitted by the crack. In the absence of dissipative (viscous) stresses, standard thermodynamic arguments $[13,14]$ lead to the state equations

$$
\begin{aligned}
& \mathbf{t}=\frac{\partial \psi}{\partial \mathbf{e}}=\frac{1}{\gamma} \hat{\mathbf{D}} \cdot \mathbf{e} \\
& \Gamma=-\frac{\partial \psi}{\partial \gamma}=\frac{1}{2 \gamma^{2}} \mathbf{e} \cdot \hat{\mathbf{D}} \cdot \mathbf{e}
\end{aligned}
$$


where $\Gamma$ is the dissipative thermodynamic force associated with $\gamma$. The dissipation inequality (3) now reads

$$
\mathscr{D} \equiv \Gamma \dot{\gamma} \geqslant 0
$$

The reference stiffness $\hat{\mathbf{D}}$ is assumed to be positive definite, so that the thermodynamic force $\Gamma$ defined by Equation (5) is always non-negative. Condition (6) then implies that the rate of $\gamma$ must not be negative, i.e. that $\gamma$ can only increase or remain constant but can never decrease. Symmetry arguments lead to the condition that, in local co-ordinates for which the first axis is aligned with the crack normal $\mathbf{n}$, the reference stiffness must be represented by a diagonal matrix

$$
\hat{\mathbf{D}}=\left[\begin{array}{ccc}
D_{n n} & 0 & 0 \\
0 & D_{s s} & 0 \\
0 & 0 & D_{s s}
\end{array}\right]
$$

because, e.g. normal opening of the crack should not generate shear tractions on its faces, and sliding in a given tangential direction should not produce shear tractions in the perpendicular direction (unless the material is anisotropic). Of course, slip in the crack can affect the normal traction (due to dilatancy), but this phenomenon is out of scope of the present damage-based model and it would have to be incorporated into a generalized version of the model through an additional inelastic strain. In view of Equation (7), the traction-separation law (4) can be written as

$$
\begin{aligned}
t_{n} & =\frac{D_{n n}}{\gamma} e_{n} \\
\mathbf{t}_{s} & =\frac{D_{s s}}{\gamma} \mathbf{e}_{s}
\end{aligned}
$$

where $t_{n}=\mathbf{t} \cdot \mathbf{n}$ is the normal traction transmitted by the crack, $\mathbf{t}_{s}=\mathbf{t}-t_{n} \mathbf{n}$ is the tangential traction, $e_{n}=\mathbf{e} \cdot \mathbf{n}$ is the normal component of the separation vector (crack opening), and $\mathbf{e}_{s}=\mathbf{e}-e_{n} \mathbf{n}$ is the tangential component of the separation vector (crack sliding).

To complete the theory, it is necessary to postulate an evolution law for the compliance parameter, $\gamma$. Using the formalism of generalized standard materials [13], we assume the existence of a (dual) dissipation potential $\phi^{*}(\Gamma, \gamma)$ such that

$$
\dot{\gamma}=\frac{\partial \phi^{*}(\Gamma, \gamma)}{\partial \Gamma}
$$

If the potential is nonnegative, equal to zero for $\Gamma=0$, and convex with respect to the thermodynamic force $\Gamma$ (for any admissible value of the internal variable $\gamma$ ), the dissipation

$$
\mathscr{D} \equiv \Gamma \dot{\gamma}=\Gamma \frac{\partial \phi^{*}}{\partial \Gamma}
$$

is guaranteed to be non-negative. Now it is necessary to specify the dissipation potential. In analogy to continuum damage models, let us define a loading function $f^{*}(\Gamma, \gamma)$ such that the inequality $f^{*}<0$ characterizes the elastic domain. If $f^{*}<0$, the deformation process is reversible (elastic), i.e. the dissipation rate must be zero. If $f^{*}=0$, damage grows, which is accompanied by energy 
dissipation. For rate-independent models the damage increment is assumed to be instantaneous, and states for which $f^{*}>0$ can never be reached. All this is reflected by a dissipation potential $\phi^{*}(\Gamma, \gamma)$ defined as the indicator function [14] of the elastic domain $\mathscr{F}=\left\{(\Gamma, \gamma) \mid f^{*}(\Gamma, \gamma) \leqslant 0\right\}$. The indicator function is equal to zero in $\mathscr{F}$ and equal to infinity outside $\mathscr{F}$. Its gradient with respect to $\Gamma$ that appears in Equation (10) must be interpreted in the sense of a subdifferential [14]. The resulting evolution law is described by

$$
\dot{\gamma}=\dot{\lambda} \frac{\partial f^{*}(\Gamma, \gamma)}{\partial \Gamma}
$$

along with the Kuhn-Tucker conditions,

$$
\dot{\lambda} \geqslant 0, \quad f^{*} \leqslant 0, \quad \dot{\lambda} f^{*}=0
$$

from which we can derive the consistency condition,

$$
\dot{\lambda} \dot{f}^{*}=0
$$

Equations (13) and (14) are formally the same as for standard plasticity, where $f^{*}$ would be the yield function and $\lambda$ would be the plastic multiplier. The response of the model is fully determined by specifying the loading function, $f^{*}$. To comply with the thermodynamic formalism, we have introduced $f^{*}$ as a function of the internal variable $\gamma$ and the associated thermodynamic force $\Gamma$. However, when constructing the specific form of the loading function it is convenient to work with variables which have a direct physical meaning. Inspecting the state law (5) we observe that the force $\Gamma$ is related to $\mathbf{e} \cdot \hat{\mathbf{D}} \cdot \mathbf{e}$, which can be interpreted as a scalar measure of the displacement jump across the discontinuity. The state law can be rewritten as

$$
\Gamma=\frac{D_{n n}}{2 \gamma^{2}}\left(e_{n}^{2}+\frac{D_{s s}}{D_{n n}} e_{s}^{2}\right)=\frac{D_{n n} e^{2}}{2 \gamma^{2}}
$$

where $e_{s}=\left\|\mathbf{e}_{s}\right\|$ is the Euclidean norm of the tangential component of the separation vector (crack sliding), and

$$
\tilde{e}=\sqrt{e_{n}^{2}+\frac{D_{s s}}{D_{n n}} e_{s}^{2}}=\sqrt{\frac{\mathbf{e} \cdot \hat{\mathbf{D}} \cdot \mathbf{e}}{D_{n n}}}
$$

can be called the equivalent separation (in analogy to the equivalent strain in continuum damage mechanics).

Suppose that the traction-separation curve for fracture under pure Mode I has been identified from experiments and described by an explicit relation $t_{n}=g\left(e_{n}\right)$. This relation should be reproduced by the constitutive law (8), which means that the compliance parameter under monotonically increasing crack opening must satisfy the relation $\gamma=F\left(e_{n}\right)$ where

$$
F\left(e_{n}\right)=D_{n n} e_{n} / g\left(e_{n}\right)
$$

Note that, for decreasing functions $g\left(e_{n}\right)$ that characterize softening, $F\left(e_{n}\right)$ is always an increasing function. This is closely related to the fact that the compliance parameter (inversely proportional to the slope of the line connecting the current point on the traction-separation curve with the origin) is monotonically increasing as long as the crack opening $e_{n}$ keeps growing. If $e_{n}$ drops 
below its maximum previously reached value, unloading takes place and the compliance parameter is temporarily frozen. The elastic domain is therefore characterized by the condition

$$
f\left(e_{n}, \gamma\right) \equiv F\left(e_{n}\right)-\gamma \leqslant 0
$$

For the special loading path under consideration we have $\tilde{e}=e_{n}$ and from Equation (15) it follows that $\Gamma=D_{n n} e_{n}^{2} / 2 \gamma^{2}$. Substituting $e_{n}=\gamma \sqrt{2 \Gamma / D_{n n}}$ into (18) we obtain the expression for the loading function in terms of variables $\Gamma$ and $\gamma$,

$$
f^{*}(\Gamma, \gamma) \equiv F\left(\gamma \sqrt{\frac{2 \Gamma}{D_{n n}}}\right)-\gamma
$$

This extends the description of the elastic domain to a general mixed-mode situation, based on the assumption that the evolution of damage is driven by the thermodynamic force $\Gamma$ associated with the compliance parameter $\gamma$. For the actual numerical implementation it is more convenient to deal with the loading condition expressed in terms of the equivalent separation,

$$
f(\tilde{e}, \gamma) \equiv F(\tilde{e})-\gamma \leqslant 0
$$

Since $F$ is an increasing function, the partial derivative $\partial f^{*} / \partial \Gamma$ is always positive, and so $\dot{\lambda}$ and $\dot{\gamma}$ from Equation (12) always have the same sign. Moreover, loading functions $f$ and $f^{*}$ always have the same value (for arguments corresponding to the same state). Consequently, the Kuhn-Tucker conditions (13) can be replaced by equivalent conditions

$$
\dot{\gamma} \geqslant 0, \quad f \leqslant 0, \quad \dot{\gamma} f=0
$$

As an example, consider the exponential traction-separation law,

$$
t_{n}=g\left(e_{n}\right) \equiv f_{\mathrm{t}} \exp \left(-\frac{e_{n}}{e_{f}}\right)
$$

where $f_{\mathrm{t}}$ is the tensile strength and $e_{f}=G_{f} / f_{\mathrm{t}}, G_{f}$ being the Mode-I fracture energy. The corresponding loading function is constructed as

$$
f(\tilde{e}, \gamma)=\frac{D_{n n} \tilde{e}}{f_{\mathrm{t}}} \exp \left(\frac{\tilde{e}}{e_{f}}\right)-\gamma
$$

The evolution of normal traction $t_{n}$, compliance parameter $\gamma$ and thermodynamic force $\Gamma$ during monotonic loading under pure Mode I, is depicted in Figure 1.

\subsection{Crack closure}

So far we have considered only cracks that are opening or partially closing. The model can be improved by taking into account the unilateral character of damage manifested as a stiffness recovery after a complete crack closure. The subsequent developments could be cast into the thermodynamic framework, similar to Reference [15]. For the sake of clarity we present an alternative approach, based on a direct formulation of the state and evolution laws.

It is clear that the crack faces cannot overlap, and so the normal component of the separation vector should never become negative. At the moment when the normal separation vanishes, the crack faces establish contact and become capable of transmitting compressive tractions without a 
(a)

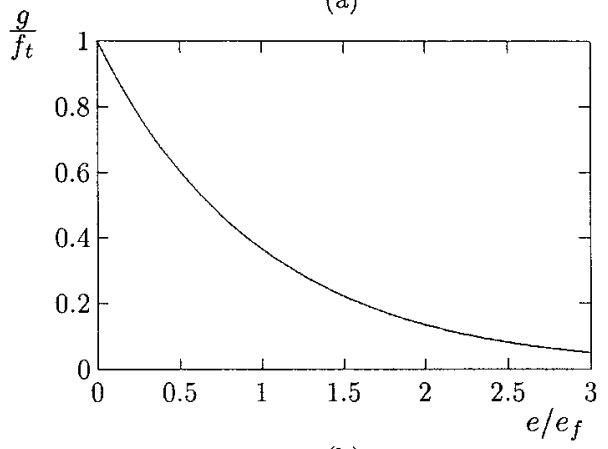

(b)

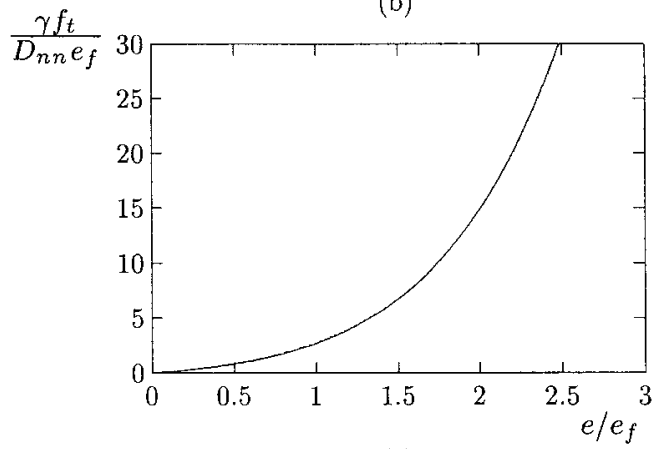

(c)

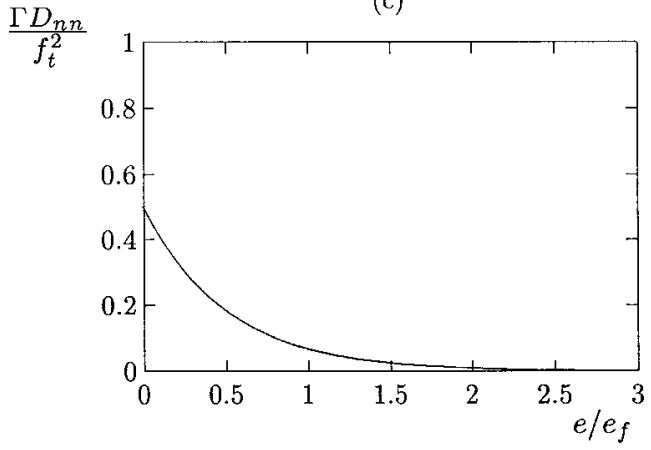

Figure 1. Dimensionless representation of the evolution of: (a) normal traction $t_{n}=g\left(e_{n}\right)$;

(b) compliance parameter $\gamma$; (c) thermodynamic force $\Gamma$.

further change of the normal displacement jump, $e_{n}$. Note that the normal part of the tractionseparation law (8) describes only the case when $e_{n}>0$. Upon crack closure, it has to be replaced by conditions $e_{n}=0$ and $t_{n} \leqslant 0$. Both cases are simultaneously covered by conditions

$$
t_{n}-\frac{D_{n n}}{\gamma} e_{n} \leqslant 0, \quad e_{n} \geqslant 0, \quad\left(t_{n}-\frac{D_{n n}}{\gamma} e_{n}\right) e_{n}=0
$$

having again the Kuhn-Tucker form. For a closed crack $\left(e_{n}=0\right)$, it is necessary to modify Equation (9) governing the evolution of the sliding components of the displacement jump vector. 
Sliding can take place even if the crack is closed, provided that the shear traction is sufficiently large to overcome the residual cohesion of the crack augmented by mobilized dry friction. For simplicity, consider first a two-dimensional model with a line crack, for which the traction and separation vectors have only one shear component, respectively denoted as $t_{s}$ and $e_{s}$. The residual cohesive resistance in shear is the shear traction computed from the damage model (9),

$$
t_{s}^{(\mathrm{coh})}=\frac{D_{s s}}{\gamma} e_{s}
$$

In the presence of a compressive normal traction, $t_{n}<0$, the maximum shear traction that can be transmitted by friction is

$$
t_{s}^{(\text {fric })}=\mu\left|t_{n}\right|=-\mu t_{n}
$$

where $\mu$ is the coefficient of friction between the cracked surfaces. Equation (26) can be reformulated as

$$
t_{s}^{(\text {fric })}=\mu\left\langle-t_{n}\right\rangle
$$

where $\langle$.$\rangle are the McAuley brackets ('positive part of'). For scalars, the positive part is defined$ as $\langle x\rangle=\max (0, x)$. Relation (27) remains valid even in the separation mode, when $t_{n}>0$ and $\left\langle-t_{n}\right\rangle=0$.

If the actual shear traction deviates from the cohesive shear traction $t_{s}^{(\mathrm{coh})}$ by less than $t_{s}^{\text {(fric) }}$, sliding does not occur and the sliding discontinuity $e_{s}$ remains constant. Once the critical value of $t_{s}$ is reached, sliding starts in the direction determined by the sign of $t_{s}-t_{s}^{(\text {coh })}$. Introducing a new loading function,

$$
f_{s}\left(\mathbf{t}, e_{s}, \gamma\right)=\left|t_{s}-\frac{D_{s s}}{\gamma} e_{s}\right|-\mu\left\langle-t_{n}\right\rangle
$$

and a 'slip multiplier', $\lambda_{s}$, we can describe the present model by the 'slip rule'

$$
\dot{e}_{s}=\dot{\lambda}_{s} \frac{\partial f_{s}}{\partial t_{s}}
$$

and the loading/unloading conditions

$$
f_{s} \leqslant 0, \quad \dot{\lambda}_{s} \geqslant 0, \quad f_{s} \dot{\lambda}_{s}=0
$$

During sliding, the loading function $f_{s}$ must remain equal to zero, and so $\dot{f}_{s}=0$. Differentiating (28) we obtain the consistency condition

$$
\left(\dot{t}_{s}+\frac{D_{s s} e_{s}}{\gamma^{2}} \dot{\gamma}-\frac{D_{s s}}{\gamma} \dot{e}_{s}\right) \operatorname{sgn}\left(t_{s}-\frac{D_{s s} e_{s}}{\gamma}\right)+\mu \dot{t}_{n} H\left(-t_{n}\right)=0
$$

where $H($.$) denotes the Heaviside function. During the sliding process the crack can experience$ further damage, which is reflected in Equation (31) by the term with $\dot{\gamma}$.

Generalization to a three-dimensional model with a crack represented by an internal surface is straightforward. Scalar shear components $t_{s}$ and $e_{s}$ are replaced by vectors $\mathbf{t}_{s}$ and $\mathbf{e}_{s}$, and the loading function (28) is reformulated as

$$
f_{s}\left(\mathbf{t}, \mathbf{e}_{s}, \gamma\right)=\left\|\mathbf{t}_{s}-\frac{D_{s s}}{\gamma} \mathbf{e}_{s}\right\|-\mu\left\langle-t_{n}\right\rangle
$$


The slip rule (29) is generalized to

$$
\dot{\mathbf{e}}_{s}=\dot{\lambda}_{s} \frac{\partial f_{s}}{\partial \mathbf{t}_{s}}
$$

and the consistency condition (31) reads

$$
\left(\dot{\mathbf{t}}_{s}+\frac{D_{s s} \mathbf{e}_{s}}{\gamma^{2}} \dot{\gamma}-\frac{D_{s S}}{\gamma} \dot{\mathbf{e}}_{s}\right) \cdot \overline{\boldsymbol{v}}+\mu \dot{t}_{n} H\left(-t_{n}\right)=0
$$

where

$$
\overline{\boldsymbol{v}}=\frac{\mathbf{t}_{s}-\frac{D_{s s}}{\gamma} \mathbf{e}_{s}}{\left\|\mathbf{t}_{s}-\frac{D_{s s}}{\gamma} \mathbf{e}_{s}\right\|}
$$

\subsection{Comparison with other models}

It is interesting to compare the present formulation with other constitutive models relating displacement jumps to tractions transmitted across a discontinuity surface or line. Phenomena such as debonding, delamination, or intergranular damage in composites and metals are often described by cohesive zone models, going back to the ideas of Dugdale [16] and Barenblatt [17] and recently developed in a modern computational framework by Needleman [18], Tvergaard and Hutchinson $[19,20]$, and Ortiz and coworkers [21-23]. Needleman's original model is based on an elastic potential without any internal variables, i.e. it has the character of a non-linear elastic model with a path-independent work of separation. Such simple approach is suitable only if the crack opening grows monotonically, but it fails to give reasonable results for (even partially) closing cracks. Moreover, the model has a non-zero initial compliance, which means that the displacement jump starts growing whenever non-zero tractions are applied, no matter how small they are. This is appropriate for simulations of pre-existing material interfaces (grain boundaries, matrix-inclusion interfaces, etc.) with a well-defined geometrical structure that can be taken into account by the finite element mesh. When the aim is to simulate a crack propagating along an arbitrary path that is not known in advance, potential surfaces (or lines, in two dimensions) of decohesion must be interspersed throughout the material [24]. Their high initial stiffness has an adverse effect on the conditioning of the global stiffness for implicit methods, or on the critical time step for explicit methods. Moreover, due to the finite number of potential discontinuity segments, the crack propagation path is locally always constrained to a discrete set of directions, typically spaced by $45^{\circ}$ or $60^{\circ}$. The present paper advocates the concept of discontinuities inserted (at the right place and with the correct orientation) when the stresses reach a certain critical level, which is certainly a more flexible approach.

A cohesive zone model with a non-zero initial compliance was also used by Tvergaard and Hutchinson [20] and by Wei and Hutchinson [25], who studied the interplay between plastic yielding in a small process zone and separation processes at an interface between two materials. They introduced a dimensionless crack separation measure $\lambda$ that is closely related to the equivalent separation $\tilde{e}$ used in the present paper. In fact, $\lambda$ can be interpreted as the ratio $\tilde{e} / e_{f}$ where $e_{f}$ is a characteristic value of separation. Tvergaard and Hutchinson worked with a trilinear tractionseparation law, for which the traction transmitted by the discontinuity completely vanishes at a finite value of separation. This naturally sets the length scale of the model, and $e_{f}$ is in this case 
the value of equivalent separation at which the transmitted tractions vanish. For more general laws with an asymptotic decay of the cohesive tractions, the characteristic length can be defined, e.g. as the separation energy divided by strength (this is the meaning of $e_{f}$ in the exponential law (22)).

Ortiz [26] suggested a derivation of the traction-separation law for Mode I. from a micromechanical model based on an array of collinear microcracks. Camacho and Ortiz [21] studied fracture and fragmentation in brittle materials using a cohesive model with possible crack initiation under mixed mode. The stress-separation law was formulated for two distinct regimes, called tension and compression. However, it seems that the model was not fully consistent. For example, in the tensile regime the magnitude of shear traction depended only on the normal separation, and the sliding component of separation affected only the sign of the shear traction. This would mean that (i) the interface does not degrade if the opening remains constant and only the sliding component increases, and (ii) the shear traction jumps from a positive value to a negative one or vice versa when the sliding component of separation changes sign. After the submission of the present manuscript, Ortiz and coworkers published new papers in which they use a similar thermodynamic framework [22] and extend it to the area of finite opening displacements [23]. They work with the notion of an effective opening displacement, which is in fact identical with the equivalent separation $\tilde{e}$ from the present paper.

Cohesive zone models are of course intimately related to the fictitious crack model of Hillerborg [27]. Early applications in the area of concrete fracture were limited to Mode-I. situations. An extension to cracks and interfaces opening under mixed-mode conditions was developed, e.g. by Cervenka [28], who exploited a loading function in the space of normal and shear tractions, originally proposed by Carol and Prat [29] in the context of statically constrained microplane models. Červenka's model requires a non-zero compliance of the interface before the actual crack initiation, and therefore is not suitable for discontinuities embedded in finite elements. The traction-separation laws for embedded discontinuities are usually postulated in a plasticity format [7, 10,30-33] or constructed as extensions of the cohesive crack model to mixed-mode situations [4-6] using concepts similar to fixed crack versions of smeared crack models. However, plasticity-based models do not properly describe unloading of a brittle material. This problem becomes especially severe at late stages of the degradation process when the crack is stress free and, according to the plasticity theory, a reversal of the opening rate immediately generates a compressive traction, which is not physical. Models inspired by the fictitious crack theory are close to interface damage mechanics $[7,10,34]$, which provides a natural description of the gradual loss of integrity. In this framework, a difficult issue is a proper treatment of stiffness recovery upon complete crack closure with possible frictional sliding. Such effects were consistently taken into account by Cangemi et al. [15] and by Chaboche and coworkers $[35,36]$ in the context of interface models for delamination and debonding of fiber-matrix composites. For cracks, virtually the same approach was proposed in the internal report [37] that served as a basis of the present paper.

\section{ELEMENT WITH EMBEDDED DISPLACEMENT DISCONTINUITY}

As already alluded to in the Introduction, the kinematic representation of highly localized fracture can be substantially improved by incorporating displacement discontinuities into the finite element interpolation. A historical overview and a classification of various approaches from the literature are available in Reference [12]. It is shown there that the optimal performance is achieved if the kinematic and static equations are constructed independently, based on their physical meaning. 

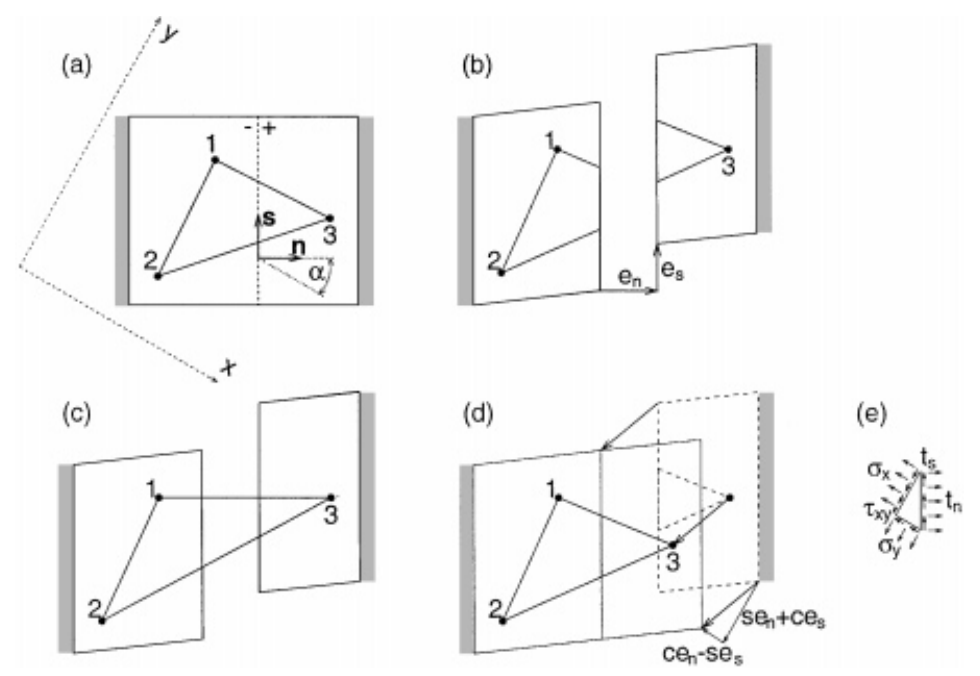

Figure 2. CST element with an embedded displacement discontinuity: (a) global co-ordinate system $x, y$ and local co-ordinate system $n, s$ aligned with the crack; (b) normal and tangential component of the displacement jump; (c) representation of crack by equivalent strain smeared over the finite element; (d) element deformation due to strain in the bulk material, with the contribution of crack opening and sliding to nodal displacements subtracted; (e) equilibrium between tractions across the crack and stresses in the bulk material.

This so-called statically and kinematically optimal nonsymmetric formulation (SKON) shall be adopted here.

To the author's best knowledge, the first publication combining the 'optimal' static and kinematic equations is due to Dvorkin et al. [4], even though this aspect was not particularly emphasized in that paper. A very similar element was constructed by Klisinski et al. [5], based on simple and instructive physical considerations. In a later paper [8], the same technique was applied to a constant-strain triangle. A general version of the SKON formulation for an arbitrary type of parent element was proposed in a short paper by Simo and Oliver [7] and fully described by Oliver [10].

In the present paper, the damage-based constitutive formulation from Section 2 shall be implemented into the simple triangular element proposed by Olofsson et al. [8]. For simplicity we assume that the bulk material surrounding the discontinuity remains linear elastic and that the crack initiation is controled by the Rankine criterion of maximum principal stress.

The basic idea is that the displacement field is decomposed into a continuous part and a discontinuous part due to the opening and sliding of a crack; see Figure 2(b). The same decomposition applies to the nodal displacements of a finite element. Instead of smearing the displacement jump over the area of the element and replacing it by an equivalent inelastic strain, as is done by standard smeared crack models (Figure 2(c)), we represent the discontinuity by additional degrees of freedom collected in a column matrix e. The effect of crack opening and sliding is then subtracted from the nodal displacement vector, $\mathbf{d}=\left\{u_{1}, v_{1}, u_{2}, v_{2}, u_{3}, v_{3}\right\}^{\mathrm{T}}$, and only the nodal displacements due to the continuous deformation serve as the input for the evaluation of strains in the bulk material, $\boldsymbol{\varepsilon}$; see Figure 2(d). This leads to the kinematic equations in the form

$$
\varepsilon=\mathbf{B}(\mathbf{d}-\mathbf{H e})
$$


where $\mathbf{B}$ is the standard strain-displacement matrix, and $\mathbf{H}$ is a matrix reflecting the effect of crack opening on the nodal displacements. In the context of finite elements we make use of the engineering notation, i.e. the bold Greek letters $\boldsymbol{\sigma}$ and $\boldsymbol{\varepsilon}$ now denote column matrices $\boldsymbol{\sigma}=\left\{\sigma_{x}, \sigma_{y}, \tau_{x y}\right\}^{\mathrm{T}}$ and $\boldsymbol{\varepsilon}=\left\{\varepsilon_{x}, \varepsilon_{y}, \gamma_{x y}\right\}^{\mathrm{T}}$ instead of second-order tensors.

In general, the displacement jump is approximated by a suitable function, for example a polynomial one. It is easy to show that the approximation need not be continuous. For triangular elements with linear displacement interpolation, the strains and stresses in the bulk are constant in each element, and so it is natural to approximate the displacement jump also by a piecewise constant function. In each element, the jump is described by its normal (opening) component, $e_{n}$, and tangential (sliding) component, $e_{s}$; see Figure 2(b). These additional degrees of freedom have an internal character and can be eliminated on the element level, which means that the global equilibrium equations are written exclusively in terms of the standard unknowns - nodal displacements. From Figure 2(d) it is clear that the crack-effect matrix is given by

$$
\mathbf{H}=\left[\begin{array}{cc}
0 & 0 \\
0 & 0 \\
0 & 0 \\
0 & 0 \\
c & -s \\
s & c
\end{array}\right]
$$

provided that the discontinuity line separates node 3 from nodes 1 and 2 (in local numbering). In Equation (37), $c=\cos \alpha$ and $s=\sin \alpha$, where $\alpha$ is the angle between the normal to the crack and the global $x$-axis; see Figure 2(a).

Strains in the bulk material generate certain stresses, $\boldsymbol{\sigma}$, which are here computed from the equations of linear elasticity,

$$
\boldsymbol{\sigma}=\mathbf{D}_{e} \boldsymbol{\varepsilon}
$$

but in general the constitutive law for the bulk material could be non-linear. The tractions transmitted by the crack (damaging surface), $\mathbf{t}$, can be computed from the displacement jump using the traction-separation law from the preceding section. Recall the constitutive relation for an opening or partially closing crack,

$$
\mathbf{t}=\frac{1}{\gamma} \hat{\mathbf{D}} \mathbf{e}
$$

and the evolution law for the compliance parameter, described by the loading/unloading conditions (21).

The stresses in the bulk and the tractions across the crack must satisfy certain conditions that express internal equilibrium and serve as static equations associated with the internal degrees of freedom, e. The most natural requirement is that the traction vector be equal to the stress tensor contracted with the crack normal, similar to static boundary conditions. This internal equilibrium (traction continuity) condition can be derived from equilibrium of an elementary triangle with one side on the discontinuity line; see Figure 2(e). In the engineering notation the traction continuity condition reads

$$
\mathbf{P}^{\mathrm{T}} \boldsymbol{\sigma}=\mathbf{t}
$$




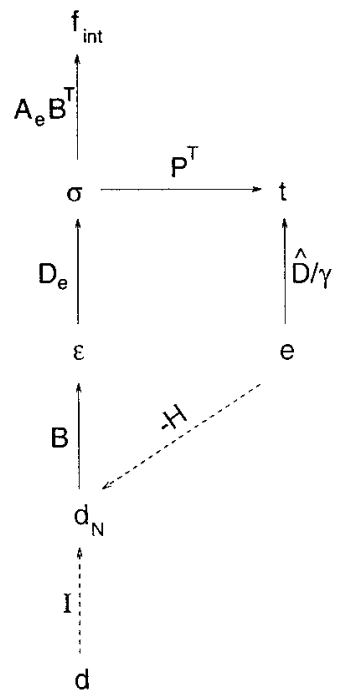

Figure 3. Structure of the equations describing the CST element with an embedded displacement discontinuity (SKON formulation).

where

$$
\mathbf{P}=\left[\begin{array}{cc}
c^{2} & -c s \\
s^{2} & c s \\
2 c s & c^{2}-s^{2}
\end{array}\right]
$$

is a stress rotation matrix. For linear triangles, both $\mathbf{t}$ and $\boldsymbol{\sigma}$ are constant in each element, and so condition (40) can be satisfied exactly. In general, it would have to be enforced in a weak sense.

Finally, the nodal forces are evaluated from the standard relation

$$
\mathbf{f}_{\text {int }}=\int_{A_{e}} \mathbf{B}^{\mathrm{T}} \boldsymbol{\sigma} \mathrm{d} A=A_{e} \mathbf{B}^{\mathrm{T}} \boldsymbol{\sigma}
$$

where $A_{e}$ is the area of the element.

The structure of the basic equations describing a CST element with an embedded displacement discontinuity is schematically depicted in Figure 3. Each arrow leads from a certain 'source' to a 'receiver' and is associated with a transformation of the source. Dashed arrows indicate that the transformed source is added to the receiver, while solid arrows mean that the transformed source is equal to the receiver.

Substituting into the traction continuity condition (40) Equations (38) and (36), we obtain a useful expression for the traction vector in terms of the kinematic variables,

$$
\mathbf{t}=\mathbf{P}^{\mathrm{T}} \mathbf{D}_{e} \mathbf{B}(\mathbf{d}-\mathbf{H e})=\mathbf{A}(\mathbf{d}-\mathbf{H e})
$$

where we have denoted

$$
\mathbf{A}=\mathbf{P}^{\mathrm{T}} \mathbf{D}_{e} \mathbf{B}
$$


Comparing Equation (43) with Equation (39), we obtain after simple manipulations

$$
(\hat{\mathbf{D}}+\gamma \mathbf{A H}) \mathbf{e}-\gamma \mathbf{A d}=\mathbf{0}
$$

Combined with the evolution equation for the compliance parameter $\gamma$, Equation (45) makes it possible to express the separation vector in terms of the nodal displacements and eliminate it from the formulation.

\section{NUMERICAL PROCEDURE}

In this section, we will develop numerical algorithms needed for the implementation of a CST element with an embedded crack (alias strong discontinuity). In Sections 4.1-4.3 we restrict our attention to the case of an opening or partially closing crack. Numerical treatment of a closed crack will be discussed in Section 4.4 .

\subsection{Algorithm for evaluation of internal forces}

Suppose that the values of all variables at the end of a certain computational step number $n$ are prescribed, and our task is to calculate their values at the end of the subsequent step number $n+1$. The value after a step will be labelled by a superscript with the step number in parentheses. The approximations of nodal displacements $\mathbf{d}^{(n+1)}$ are supplied by the iterative solution of the global equilibrium equations, and during each evaluation of the element nodal forces they can be considered as given. Before crack initiation, it suffices to compute the stresses from the equations valid for linear elasticity,

$$
\boldsymbol{\sigma}^{(n+1)}=\mathbf{D}_{e} \mathbf{B d}^{(n+1)}
$$

and then check the crack initiation condition, formulated here as the simple Rankine criterion, $\sigma_{1}=f_{\mathrm{t}}$, where $\sigma_{1}$ is the maximum principal stress and $f_{\mathrm{t}}$ is the tensile strength. The direction of maximum principal stress at crack initiation provides the normal to the discontinuity, $\mathbf{n}$, and also determines the matrices $\mathbf{P}$ and $\mathbf{H}$ that depend on the crack orientation.

After crack initiation, relations (45) written at the end of step $n+1$ contain only the crack opening parameters $\mathbf{e}^{(n+1)}$ and the compliance parameter $\gamma^{(n+1)}$ as basic unknowns and, when supplemented by the loading/unloading conditions (21), they provide a sufficient number of equations for the determination of the unknowns. Let us summarize the conditions that must be satisfied at the end of the step:

$$
\begin{array}{r}
\left(\hat{\mathbf{D}}+\gamma^{(n+1)} \mathbf{A H}\right) \mathbf{e}^{(n+1)}-\gamma^{(n+1)} \mathbf{A} \mathbf{d}^{(n+1)}=\mathbf{0} \\
f\left(\tilde{e}\left(\mathbf{e}^{(n+1)}\right), \gamma^{(n+1)}\right) \leqslant 0 \\
\gamma^{(n+1)}-\gamma^{(n)} \geqslant 0 \\
\left(\gamma^{(n+1)}-\gamma^{(n)}\right) f\left(\tilde{e}\left(\mathbf{e}^{(n+1)}\right), \gamma^{(n+1)}\right)=0
\end{array}
$$

As in standard stress return algorithms of computational plasticity, we first assume that the process is reversible, i.e., the compliance parameter does not grow. This means that we tentatively set 
$\gamma^{(n+1)}=\gamma^{(n)}$ and compute $\mathbf{e}^{(n+1)}$ from Equation (47). If the solution satisfies condition (48) it is admissible, otherwise the compliance parameter must grow and condition (48) must be satisfied with an equality sign. In that case the algorithm continues by an iterative procedure, exploiting the trial values from the elastic step as initial approximations of $\mathbf{e}^{(n+1)}$ and $\gamma^{(n+1)}$. When using the Newton-Raphson solution technique, we linearize the governing equations around the most recent approximation of the solution and then solve for the corrections of the unknown variables. Denoting the current approximations by $\overline{\mathbf{e}}$ and $\bar{\gamma}$ and their corrections by $\Delta \mathbf{e}$ and $\Delta \gamma$, and taking into account the special form of the loading function (20), we can write the linearized equations as

$$
\begin{aligned}
(\hat{\mathbf{D}}+\bar{\gamma} \mathbf{A H}) \Delta \mathbf{e}+\left(\mathbf{A H} \overline{\mathbf{e}}-\mathbf{A d}^{(n+1)}\right) \Delta \gamma & =\bar{\gamma} \mathbf{A d}{ }^{(n+1)}-(\hat{\mathbf{D}}+\bar{\gamma} \mathbf{A H}) \overline{\mathbf{e}} \\
\overline{\mathbf{f}}^{\mathrm{T}} \Delta \mathbf{e}-\Delta \gamma & =\bar{\gamma}-\bar{F}
\end{aligned}
$$

where $\bar{F}=F(\tilde{e}(\overline{\mathbf{e}}))$ and

$$
\overline{\mathbf{f}}=\frac{\partial F}{\partial \mathbf{e}}=\frac{\mathrm{d} F}{\mathrm{~d} \tilde{e}} \frac{\partial \tilde{e}}{\partial \mathbf{e}}
$$

evaluated at $\mathbf{e}=\overline{\mathbf{e}}$. Using Equation (52), the correction $\Delta \gamma$ can be conveniently expressed in terms of $\Delta \mathbf{e}$ and subsequently eliminated from Equation (51). Alternatively, one can replace $\gamma^{(n+1)}$ in Equation (47) by $F\left(\tilde{e}\left(\mathbf{e}^{(n+1)}\right)\right)$ and then linearize with respect to e. Both approaches lead to the same equation,

$$
\left[\hat{\mathbf{D}}+\bar{\gamma} \mathbf{A H}+\mathbf{A}\left(\mathbf{H} \overline{\mathbf{e}}-\mathbf{d}^{(n+1)}\right) \overline{\mathbf{f}}^{\mathrm{T}}\right] \Delta \mathbf{e}=\bar{\gamma} \mathbf{A}\left(\mathbf{d}^{(n+1)}-\mathbf{H} \overline{\mathbf{e}}\right)-\hat{\mathbf{D}} \overline{\mathbf{e}}
$$

where $\bar{\gamma}=F(\tilde{e}(\overline{\mathbf{e}}))$, so that the equation $f(\tilde{e}(\overline{\mathbf{e}}), \bar{\gamma}) \equiv F(\tilde{e}(\overline{\mathbf{e}}))-\bar{\gamma}=0$ is satisfied identically. Note the physical meaning of certain terms in Equation (54). The vector $\mathbf{A}\left(\mathbf{d}^{(n+1)}-\mathbf{H} \overline{\mathbf{e}}\right)=\overline{\mathbf{t}}_{\sigma}$ is the traction vector evaluated by projecting the bulk stresses while $\hat{\mathbf{D}} \overline{\mathbf{e}} / \bar{\gamma}=\overline{\mathbf{t}}_{e}$ is the traction vector evaluated from the traction-separation law. With this notation, Equation (54) can be rewritten as

$$
\left(\hat{\mathbf{D}}+\bar{\gamma} \mathbf{A H}-\overline{\mathbf{t}}_{\sigma} \overline{\mathbf{f}}^{\mathrm{T}}\right) \Delta \mathbf{e}=\bar{\gamma}\left(\overline{\mathbf{t}}_{\sigma}-\overline{\mathbf{t}}_{e}\right)
$$

Repeated solution of Equation (55) followed by updates $\overline{\mathbf{e}} \leftarrow \overline{\mathbf{e}}+\Delta \mathbf{e}$ and $\bar{\gamma} \leftarrow F(\tilde{e}(\overline{\mathbf{e}}))$ generates a sequence of approximations $\overline{\mathbf{e}}$ that converges to the desired value $\mathbf{e}^{(n+1)}$, unless the step is too large and the process diverges. In the case of divergence it is usually sufficient to apply the increment of the nodal displacements in several smaller substeps.

At late stages of the degradation process, the compliance parameter $\gamma$ assumes extremely large values. Robustness of the algorithm is increased if Equation (47) is divided by $\gamma^{(n+1)}$ and condition (48), which originally reads $F\left(\tilde{e}\left(\mathbf{e}^{(n+1)}\right)\right)-\gamma^{(n+1)} \leqslant 0$, is reformulated as $F^{*}\left(\tilde{e}\left(\mathbf{e}^{(n+1)}\right)\right)-1 / \gamma^{(n+1)} \geqslant 0$ where $F^{*}(\tilde{e})=1 / F(\tilde{e})$. Instead of the compliance parameter itself, we can deal with its inverse value $\gamma^{*}=1 / \gamma$, which does not blow up but tends to zero. After linearization of the governing equations we obtain

$$
\left(\bar{\gamma}^{*} \hat{\mathbf{D}}+\mathbf{A H}+\hat{\mathbf{D}} \overline{\mathbf{e}}^{* \mathrm{~T}}\right) \Delta \mathbf{e}=\mathbf{A}\left(\mathbf{d}^{(n+1)}-\mathbf{H} \overline{\mathbf{e}}\right)-F^{*}(\overline{\mathbf{e}}) \hat{\mathbf{D}} \overline{\mathbf{e}}
$$

in which $\overline{\mathbf{f}}^{*}$ is the gradient $\partial F^{*} / \partial \mathbf{e}$ evaluated at $\mathbf{e}=\overline{\mathbf{e}}$. The right-hand side of Equation (56) can be identified as the difference $\overline{\mathbf{t}}_{\sigma}-\overline{\mathbf{t}}_{e}$ between the traction vectors, respectively, evaluated by projecting 
the stresses and by applying the traction-separation law. Equation (56) is in fact Equation (55) divided by $\bar{\gamma}$, with $-\overline{\mathbf{t}}_{\sigma} \overline{\mathbf{f}}^{\mathrm{T}} / \bar{\gamma}$ replaced by $\hat{\mathbf{D}} \overline{\mathbf{e}} \overline{\mathbf{f}}^{* \mathrm{~T}}$. Since

$$
\mathbf{f}=\frac{\mathrm{d} F}{\mathrm{~d} \tilde{e}} \frac{\partial \tilde{e}}{\partial \mathbf{e}}=\frac{\mathrm{d}}{\mathrm{d} \tilde{e}}\left(\frac{1}{F^{*}}\right) \frac{\partial \tilde{e}}{\partial \mathbf{e}}=-\frac{1}{F^{* 2}} \frac{\mathrm{d} F^{*}}{\mathrm{~d} \tilde{e}} \frac{\partial \tilde{e}}{\partial \mathbf{e}}=-\gamma^{2} \mathbf{f}^{*}
$$

the matrix $-\overline{\mathbf{t}}_{\sigma} \overline{\mathbf{f}}^{\mathrm{T}} / \bar{\gamma}=\bar{\gamma}_{\sigma} \overline{\mathbf{f}}^{* \mathrm{~T}}$ differs from $\hat{\mathbf{D}} \overline{\mathbf{e}} \overline{\mathbf{f}}^{* \mathrm{~T}}=\bar{\gamma}_{e} \overline{\mathbf{f}}^{* \mathrm{~T}}$ only by the technique used for evaluation of the tractions, $\overline{\mathbf{t}}$.

Adopting the modified solution strategy based on the inverse of the compliance parameter rather than on the compliance parameter itself is especially important for models which consider the crack to be completely stress-free at a finite value of separation. For example, for a linear tractionseparation curve we have

$$
g\left(e_{n}\right)= \begin{cases}f_{\mathrm{t}}\left(1-\frac{e_{n}}{e_{f}}\right) & \text { if } e_{n} \leqslant e_{f} \\ 0 & \text { if } e_{n} \geqslant e_{f}\end{cases}
$$

where $e_{f}$ is the value of opening at which the traction completely vanishes. Consequently, the function

$$
F(\tilde{e})=\frac{D_{n n} \tilde{e}}{g(\tilde{e})}=\frac{D_{n n} e_{f}}{f_{\mathrm{t}}} \frac{\tilde{e}}{e_{f}-\tilde{e}}
$$

is properly defined only for $\tilde{e}<e_{f}$ while outside this domain it should have an infinite value. On the other hand, the reciprocal function is conveniently defined as

$$
F^{*}(\tilde{e})=\frac{g(\tilde{e})}{D_{n n} \tilde{e}}= \begin{cases}\frac{f_{\mathrm{t}}}{D_{n n} e_{f}} \frac{e_{f}-\tilde{e}}{\tilde{e}} & \text { if } 0<\tilde{e} \leqslant e_{f} \\ 0 & \text { if } e_{f} \leqslant \tilde{e}\end{cases}
$$

The gradient $\mathbf{f}^{*}$ remains bounded as $\tilde{e}$ approaches $e_{f}$, and it vanishes for $\tilde{e}>e_{f}$. Thus, after complete separation of the crack faces, Equation (56) reduces to

$$
\mathbf{A H} \Delta \mathbf{e}=\mathbf{A}\left(\mathbf{d}^{(n+1)}-\mathbf{H e} \overline{\mathbf{e}}\right)
$$

If the element size is below a certain critical value, the matrix $\mathbf{A H}$ is regular, and Equations (61) have a unique solution. Thus the algorithm based on Equation (56) is applicable even to a completely stress-free crack.

\subsection{Element stiffness matrix}

The tangent stiffness of an element with embedded discontinuity can be constructed by expressing the separation rate in terms of the displacement rate and substituting into the rate form of the basic equations introduced in Section 3. Differentiation of Equation (45) leads to the rate equations

$$
(\hat{\mathbf{D}}+\gamma \mathbf{A H}) \dot{\mathbf{e}}+\mathbf{A}(\mathbf{H e}-\mathbf{d}) \dot{\gamma}-\gamma \mathbf{A} \dot{\mathbf{d}}=\mathbf{0}
$$

For growing damage we have $\dot{f}=\mathbf{f}^{\mathrm{T}} \dot{\mathbf{e}}-\dot{\gamma}=0$, and substituting $\dot{\gamma}=\mathbf{f}^{\mathrm{T}} \dot{\mathbf{e}}$ into Equation (62) it is possible to express the separation rate

$$
\dot{\mathbf{e}}=\gamma\left[\hat{\mathbf{D}}+\gamma \mathbf{A H}+\mathbf{A}(\mathbf{H e}-\mathbf{d}) \mathbf{f}^{\mathrm{T}}\right]^{-1} \mathbf{A d}
$$


The rate of nodal forces is now obtained from the rate form of Equations (36), (38), and (42) as

$$
\dot{\mathbf{f}}_{\text {int }}=A_{e} \mathbf{B}^{\mathrm{T}} \mathbf{D}_{e} \mathbf{B}(\dot{\mathbf{d}}-\mathbf{H} \dot{\mathbf{e}})=\mathbf{K}_{e}\left(\mathbf{I}-\gamma \mathbf{H}\left[\hat{\mathbf{D}}+\gamma \mathbf{A} \mathbf{H}+\mathbf{A}(\mathbf{H e}-\mathbf{d}) \mathbf{f}^{\mathrm{T}}\right]^{-1} \mathbf{A}\right) \dot{\mathbf{d}}
$$

where

$$
\mathbf{K}_{e}=A_{e} \mathbf{B}^{\mathrm{T}} \mathbf{D}_{e} \mathbf{B}
$$

is the elastic element stiffness matrix, and $\mathbf{I}$ is the unit matrix. Recognizing $\mathbf{A}(\mathbf{H e}-\mathbf{d})$ as minus the traction vector, we can present the tangent element stiffness matrix in the form

$$
\mathbf{K}=\mathbf{K}_{e}-\gamma \mathbf{K}_{e} \mathbf{H}\left(\hat{\mathbf{D}}+\gamma \mathbf{A H}-\mathbf{t f}^{\mathrm{T}}\right)^{-1} \mathbf{A}
$$

An alternative expression, useful at late stages of the degradation process when the compliance parameter $\gamma$ has a large or even infinite value, would be

$$
\mathbf{K}=\mathbf{K}_{e}-\mathbf{K}_{e} \mathbf{H}\left(\gamma^{*} \hat{\mathbf{D}}+\mathbf{A} \mathbf{H}-\gamma^{*} \mathbf{t f}^{\mathrm{T}}\right)^{-1} \mathbf{A}
$$

where $\gamma^{*}=1 / \gamma$ is the inverse of the compliance parameter.

If damage does not grow (the crack is unloading), we have to repeat the derivation with $\dot{\gamma}=0$ instead of $\dot{\gamma}=\mathbf{f}^{\mathrm{T}} \dot{\mathbf{e}}$. Formulae (66) and (67), respectively, reduce to

$$
\mathbf{K}=\mathbf{K}_{e}-\gamma \mathbf{K}_{e} \mathbf{H}(\hat{\mathbf{D}}+\gamma \mathbf{A H})^{-1} \mathbf{A}
$$

and

$$
\mathbf{K}=\mathbf{K}_{e}-\mathbf{K}_{e} \mathbf{H}\left(\gamma^{*} \hat{\mathbf{D}}+\mathbf{A} \mathbf{H}\right)^{-1} \mathbf{A}
$$

For the present model, there is no difference between the stiffness matrix derived by differentiation of the constitutive equations ('continuum' stiffness) and the one derived by differentiation of the numerical algorithm ('algorithmic' or 'consistent' stiffness). The reason is that, due to the explicit nature of the damage model, the algorithm does not involve any approximation of a time rate by a finite difference expression. For the same reason, the algorithm is insensitive to the size of the applied increment, provided that the type of process (loading or unloading) remains the same.

\subsection{Singular case-onset of cracking}

Special attention should be paid to the evaluation of the element response in the first inelastic step, i.e. immediately after crack initiation. The elastic predictor gives $\overline{\mathbf{e}}=\mathbf{0}$, but at this point the gradient

$$
\mathbf{f}=\frac{\partial F}{\partial \mathbf{e}}=\frac{\mathrm{d} F}{\mathrm{~d} \tilde{e}} \frac{\partial \tilde{e}}{\partial \mathbf{e}}
$$

has a singularity. Differentiating $F(\tilde{e})=D_{n n} \tilde{e} / g(\tilde{e})$ we obtain

$$
\frac{\mathrm{d} F}{\mathrm{~d} \tilde{e}}=D_{n n} \frac{g(\tilde{e})-\tilde{e} g^{\prime}(\tilde{e})}{g^{2}(\tilde{e})}
$$


which has a finite value at $\tilde{e}=0$. Differentiation of Equation (16) written in the engineering notation leads to

$$
\frac{\partial \tilde{e}}{\partial \mathbf{e}}=\frac{\partial}{\partial \mathbf{e}} \sqrt{\frac{\mathbf{e}^{\mathrm{T}} \hat{\mathbf{D}} \mathbf{e}}{D_{n n}}}=\frac{1}{2}\left(\frac{\mathbf{e}^{\mathrm{T}} \hat{\mathbf{D}} \mathbf{e}}{D_{n n}}\right)^{-1 / 2} \frac{2 \hat{\mathbf{D}} \mathbf{e}}{D_{n n}}=\frac{\hat{\mathbf{D}} \mathbf{e}}{D_{n n} \tilde{e}}
$$

The vector $\boldsymbol{v}=\mathbf{e} / \tilde{e}$ remains bounded as e tends to the zero vector but there exists no unique limit. Nevertheless, we will show that the value of $\mathbf{f}$ for $\mathbf{e}=\mathbf{0}$, in the following denoted as $\mathbf{f}_{0}$, can be defined such that the response of the model at the instant of crack initiation remains unique.

First, note that for any $\mathbf{e} \neq \mathbf{0}$ the vector $\boldsymbol{v}$ satisfies the normalizing condition

$$
\boldsymbol{v}^{\mathrm{T}} \hat{\mathbf{D}} \boldsymbol{v}=\frac{\mathbf{e}^{\mathrm{T}} \hat{\mathbf{D}} \mathbf{e}}{\tilde{e}^{2}}=\frac{D_{n n} \tilde{e}^{2}}{\tilde{e}^{2}}=D_{n n}
$$

and that for $\mathbf{e} \rightarrow \mathbf{0}$ expression (71) tends to $D_{n n} / g(0)$. To preserve continuity, we look for $\mathbf{f}$ at $\mathbf{e}=\mathbf{0}$ in the form

$$
\mathbf{f}_{0}=\frac{\hat{\mathbf{D}} \boldsymbol{v}_{0}}{g(0)}
$$

where $\boldsymbol{v}_{0}$ is an unknown vector satisfying the normalizing condition

$$
\boldsymbol{v}_{0}^{\mathrm{T}} \hat{\mathbf{D}} \boldsymbol{v}_{0}=D_{n n}
$$

Second, consider the rate equations (62). Substituting the values at crack initiation, $\mathbf{e}=\mathbf{0}, \gamma=0$, $\dot{\gamma}=\mathbf{f}_{0}^{\mathrm{T}} \dot{\mathbf{e}}$, and $\mathbf{d}=\mathbf{d}_{0}$, we obtain a set of homogeneous linear equations

$$
\left(\hat{\mathbf{D}}-\mathbf{A d}_{0} \mathbf{f}_{0}^{\mathrm{T}}\right) \dot{\mathbf{e}}=\mathbf{0}
$$

where $\mathbf{f}_{0}$ has the form (74) and $\mathbf{A d}_{0}=\mathbf{P}^{\mathrm{T}} \mathbf{D}_{e} \mathbf{B} \mathbf{d}_{0}=\mathbf{P}^{\mathrm{T}} \boldsymbol{\sigma}_{0}=\mathbf{t}_{0}$ is the traction vector obtained by projecting the stress at crack initiation, $\boldsymbol{\sigma}_{0}$. Let us denote $\tau_{0} \equiv \mathbf{t}_{0} / g(0)$. Equations (76) have a nontrivial solution only if the matrix $\hat{\mathbf{D}}-\mathbf{A d}_{0} \mathbf{f}_{0}^{\mathrm{T}}=\hat{\mathbf{D}}-\mathbf{t}_{0} \boldsymbol{v}_{0}^{\mathrm{T}} \hat{\mathbf{D}} / g(0)=\left(\mathbf{I}-\boldsymbol{\tau}_{0} \boldsymbol{v}_{0}^{\mathrm{T}}\right) \hat{\mathbf{D}}$ is singular. Matrix $\hat{\mathbf{D}}$ is always regular and matrix $\mathbf{I}-\tau_{0} \boldsymbol{v}_{0}^{\mathrm{T}}$ is singular if and only if

$$
\tau_{0}^{\mathrm{T}} \boldsymbol{v}_{0}=1
$$

Vector $\boldsymbol{v}_{0}$, characterizing the initial direction of separation rate $\dot{\mathbf{e}}$, is the solution of Equations (75) and (77). Geometrically, Equation (75) corresponds to an ellipsoid and Equation (77) to a plane. The solution is unique if and only if the plane is tangential to the ellipsoid, which leads to the condition

$$
D_{n n} \tau_{0}^{\mathrm{T}} \hat{\mathbf{D}}^{-1} \tau_{0}=1
$$

or, equivalently,

$$
D_{n n} \mathbf{t}_{0}^{\mathrm{T}} \hat{\mathbf{D}}^{-1} \mathbf{t}_{0}=g^{2}(0)
$$

The structure of the left-hand side of Equation (79) motivates the definition of the equivalent traction,

$$
\tilde{t}=\sqrt{D_{n n} \mathbf{t}_{0}^{\mathrm{T}} \hat{\mathbf{D}}^{-1} \mathbf{t}_{0}}
$$


which is the work-conjugate quantity of the equivalent separation, $\tilde{e}$. Substituting from the constitutive relation (4) we obtain

$$
\tilde{t}=\sqrt{D_{n n} \frac{\mathbf{e}^{\mathrm{T}} \hat{\mathbf{D}}}{\gamma} \hat{\mathbf{D}}^{-1} \frac{\hat{\mathbf{D}} \mathbf{e}}{\gamma}}=\frac{D_{n n}}{\gamma} \sqrt{\frac{\mathbf{e}^{\mathrm{T}} \hat{\mathbf{D}} \mathbf{e}}{D_{n n}}}=\frac{D_{n n}}{\gamma} \tilde{e}
$$

During monotonic loading, $\gamma=F(\tilde{e})=D_{n n} \tilde{e} / g(\tilde{e})$, and so $\tilde{t}=g(\tilde{e})$. Condition (79) can now be interpreted as the requirement of consistency between the crack initiation criterion and the softening law. In the simplest version of the model, $g$ can be taken as a given function with fixed parameters, independent of mode mixity at crack initiation. In that case, $g(0)=f_{\mathrm{t}}=$ uniaxial tensile strength, and the choice of the reference stiffness $\hat{\mathbf{D}}$ immediately gives the initiation criterion, which must read

$$
D_{n n} \mathbf{t}_{0}^{\mathrm{T}} \hat{\mathbf{D}}^{-1} \mathbf{t}_{0}=f_{\mathrm{t}}^{2}
$$

Such criterion is represented by an ellipsoid in the space of tractions $\mathbf{t}$. This would be too restrictive. However, it is also possible to generalize the model and adjust the parameters that control the softening function $g$ depending on the stress state at crack initiation. The initiation criterion is then postulated independently, but the definition of $g$ must be such that $g(0)$ is equal to the equivalent traction at crack initiation, $\tilde{t}_{0}$. Such generalized model can accommodate different values of fracture energy for different fracture modes.

From the computational point of view, it is essential that condition (79) guarantees a unique solution $\boldsymbol{v}_{0}$ of Equations (75) and (77). This means that, at $\mathbf{e}=\mathbf{0}$ (crack initiation), the vector $\mathbf{f}_{0}=\hat{\mathbf{D}} \boldsymbol{v}_{0} / g(0)=D_{n n} \mathbf{t}_{0} / g^{2}(0)$ plays the role of the gradient $\mathbf{f}$. Equations (54) for the crack opening corrections $\Delta \mathbf{e}$ can now be set up even at the instant of crack initiation-we simply substitute $\mathbf{f}_{0}$ for $\overline{\mathbf{f}}$. In subsequent iterations, $\overline{\mathbf{e}}$ is already non-zero and $\overline{\mathbf{f}}$ can be evaluated from Equation (70).

\subsection{Numerical treatment of closed crack}

All numerical algorithms presented so far have considered a crack that remains at least partially open. If the model takes into account the stiffness recovery after a complete crack closure (as discussed in Section 2.2), it is necessary to check whether the converged separation vector has a positive normal component. When a negative value of $e_{n}$ is detected, the crack is closed, and a different algorithm must be applied. Most equations from Section 3 remain valid but the constitutive relation (39) must be replaced by $e_{n}=0$ and Equations (29) and (30).

Numerical treatment of a closed crack always starts from the assumption that the crack separation vector e remains constant. This corresponds to the 'sticking' mode, in which the crack surfaces are in contact and do not experience any relative motion. Setting $\overline{\mathbf{e}}=\left\{0, e_{s}^{(n)}\right\}^{\mathrm{T}}$ and $\bar{\gamma}=\gamma^{(n)}$, we can evaluate the trial tractions according to Equation (43) as

$$
\overline{\mathbf{t}}=\mathbf{A}\left(\mathbf{d}^{(n+1)}-\mathbf{H e}\right)
$$

and check the conditions

$$
\begin{array}{r}
\bar{t}_{n} \leqslant 0 \\
\left|\bar{t}_{s}-\frac{D_{s s}}{\bar{\gamma}} \bar{e}_{s}\right|-\mu\left\langle-\bar{t}_{n}\right\rangle \leqslant 0
\end{array}
$$


If both conditions are satisfied the solution is admissible, and we can accept $\overline{\mathbf{e}}$ as the value $\mathbf{e}^{(n+1)}$ at the end of the step. If the first condition is violated, the crack starts re-opening and the algorithm from Section 4.1 should be applied. If the second condition is violated, the crack starts sliding and the trial values should be corrected. At the end of the step, the loading function $f_{s}$ defined by Equation (28) must be equal to zero. It is reasonable to expect that the sign of $t_{s}-D_{s s} e_{s} / \gamma$ is correctly predicted by the trial solution that assumes no sliding, and so the condition $f_{s}\left(\mathbf{t}^{(n+1)}, e_{s}^{(n+1)}, \gamma^{(n+1)}\right)=0$ can be written as

$$
S\left(t_{s}^{(n+1)}-\frac{D_{s s}}{\gamma^{(n+1)}} e_{s}^{(n+1)}\right)+\mu t_{n}^{(n+1)}=0
$$

where

$$
S=\frac{\partial f_{s}}{\partial t_{s}}=\operatorname{sgn}\left(\bar{t}_{s}-\frac{D_{s s}}{\bar{\gamma}} \bar{e}_{s}\right)
$$

Formula (43) makes it possible to express the tractions in terms of the sliding relative displacement, $e_{s}$. The normal component of $\mathbf{e}$ is in the sliding mode equal to zero, and so we have

$$
\mathbf{t}^{(n+1)}=\mathbf{A} \mathbf{d}^{(n+1)}-\mathbf{A} \mathbf{h}_{2} e_{s}^{(n+1)}
$$

where $\mathbf{h}_{2}$ is the second column of $\mathbf{H}$. Rewriting Equation (86) as

$$
\mathbf{f}_{s}^{\mathrm{T}} \mathbf{t}^{(n+1)}-\frac{S D_{s s}}{\gamma^{(n+1)}} e_{s}^{(n+1)}=0
$$

where $\mathbf{f}_{s}=\{\mu, S\}^{\mathrm{T}}=\partial f_{s} / \partial \mathbf{t}$, and substituting from Equation (88), we obtain

$$
\mathbf{f}_{s}^{\mathrm{T}} \mathbf{A} \mathbf{d}^{(n+1)}-\left(\frac{S D_{s s}}{\gamma^{(n+1)}}+\mathbf{f}_{s}^{\mathrm{T}} \mathbf{A} \mathbf{h}_{2}\right) e_{s}^{(n+1)}=0
$$

If damage does not grow then $\gamma^{(n+1)}=\gamma^{(n)}$, and the sliding component is immediately obtained as

$$
e_{s}^{(n+1)}=\frac{\mathbf{f}_{s}^{\mathrm{T}} \mathbf{A} \mathbf{d}^{(n+1)}}{S D_{s s} / \gamma^{(n)}+\mathbf{f}_{s}^{\mathrm{T}} \mathbf{A} \mathbf{h}_{2}}
$$

However, this solution is admissible only if $F$ evaluated at $\mathbf{e}^{(n+1)}=\left\{0, e_{s}^{(n+1)}\right\}^{\mathrm{T}}$ does not exceed $\gamma^{(n)}$. Violation of this condition indicates that damage grows, and so in Equation (90) we have to consider $\gamma$ as a function of $e_{s}$. The resulting non-linear equation is then solved by the Newton method.

\section{CONCLUDING REMARKS}

Constitutive description of a damaging interface inspired by continuum damage mechanics has been proposed, and possible extensions that take into account the stiffness recovery upon crack closure and the effects of friction have been outlined. The displacement discontinuity has been incorporated into a constant-strain triangular finite element, based on the statically and kinematically optimal non-symmetric formulation. Algorithms for the evaluation of internal forces and of the element stiffness matrix have been developed and extended to special cases such as the onset of cracking, a 
completely stress-free crack, or a closed crack. Numerical examples are presented in the companion paper.

The model just presented should be understood as an initial point of departure. A number of possible improvements and refinements could be added. For example, a positive normal separation rate could be induced by the sliding process, which would reflect dilatancy due to crack tortuosity; the separation vector could have an irreversible part; or the friction coefficient could vary as the sliding process continues. However, the model formulated here reflects the most essential aspects of the behaviour of a macroscopic crack, while remaining sufficiently simple from the practical point of view.

\section{ACKNOWLEDGEMENTS}

Financial support of the Swiss Commission for Technology and Innovation under project CTI.3201.1 is gratefully acknowledged.

\section{REFERENCES}

1. Jirásek M, Zimmermann Th. Analysis of rotating crack model. Journal of Engineering Mechanics ASCE 1998; 124:842-851.

2. Ortiz M, Leroy Y, Needleman A. A finite element method for localized failure analysis. Computer Methods in Applied Mechanics and Engineering 1987; 61:189-214.

3. Belytschko T, Fish J, Engelmann BE. A finite element with embedded localization zones. Computer Methods in Applied Mechanics and Engineering 1988; 70:59-89.

4. Dvorkin EN, Cuitiño AM, Gioia G. Finite elements with displacement interpolated embedded localization lines insensitive to mesh size and distortions. International Journal for Numerical Methods in Engineering 1990; 30: $541-564$.

5. Klisinski M, Runesson K, Sture S. Finite element with inner softening band. Journal of Engineering Mechanics ASCE 1991; 117:575-587.

6. Lotfi HR. Finite element analysis of fracture in concrete and masonry structures. Ph.D. Thesis, University of Colorado, Boulder, 1992.

7. Simo JC, Oliver J. A new approach to the analysis and simulation of strain softening in solids. In Fracture and Damage in Quasibrittle Structures, Bažant ZP et al. (eds). E. \& F.N. Spon: London, 1994; 25-39.

8. Olofsson Th, Klisinski M, Nedar P. Inner softening bands: a new approach to localization in finite elements. In Computational Modelling of Concrete Structures, Mang H, Bićanić N, de Borst R (eds.) Pineridge Press: Swansea, 1994; 373-382.

9. Larsson R, Runesson K, Åkesson M. Embedded cohesive crack models based on regularized discontinuous displacements. In Fracture Mechanics of Concrete Structures, Proceedings of FraMCoS-2, Wittmann FH (ed.). Aedificatio Publishers: Freiburg, Germany, 1995; 899-911.

10. Oliver J. Modelling strong discontinuities in solid mechanics via strain softening constitutive equations. Part 1: Fundamentals. Part 2: Numerical simulation. International Journal for Numerical Methods in Engineering 1996; 39:3575-3624.

11. Sluys LJ, Berends AH. Discontinuous failure analysis for mode-I and mode-II localization problems. International Journal of Solids and Structures, to appear.

12. Jirásek M. Comparative study on finite elements with embedded cracks. Computer Methods in Applied Mechanics and Engineering, in press.

13. Lemaitre J, Chaboche J-L. Mechanics of Solid Materials. Cambridge University Press: Cambridge, UK, 1990.

14. Maugin GA. The Thermomechanics of Plasticity and Fracture. Cambridge University Press: Cambridge, UK, 1992.

15. Cangemi L, Cocu M, Raous M. Adhesion and friction model for the fiber/matrix interface of a composite. In Proceedings of the 1996 Engineering Systems Design and Analysis Conference, vol. 4, 1996; 157-163.

16. Dugdale DS. Yielding of steel sheets containing slits. Journal of the Mechanics and Physics of Solids 1960; 8:100-104.

17. Barenblatt GI. Mathematical theory of equilibrium cracks. Advances in Applied Mechanics 1962; 7:56-129.

18. Needleman A. A continuum model for void nucleation by inclusion debonding. Journal of Applied Mechanics 1987; 54:525-531.

19. Tvergaard V, Hutchinson JW. The relation between crack growth resistance and fracture process parameters in elasticplastic solids. Journal of the Mechanics and Physics of Solids 1992; 40:1377-1397. 
20. Tvergaard V, Hutchinson JW. The influence of plasticity on mixed mode interface toughness. Journal of the Mechanics and Physics of Solids 1993; 41:1119-1135.

21. Camacho GT, Ortiz M. Computational modelling of impact damage in brittle materials. International Journal of Solids and Structures 1996; 33:2899-2938.

22. Pandolfi A, Krysl P, Ortiz M. Finite element simulation of ring expansion and fragmentation: the capturing of length and time scales through cohesive models of fracture. International Journal of Fracture 1999; 95:279-297.

23. Ortiz M, Pandolfi A. Finite-deformation irreversible cohesive elements for three-dimensional crack-propagation analysis International Journal for Numerical Methods in Engineering 1999; 44:1267-1282.

24. Xu X-P, Needleman A. Numerical simulations of fast crack growth in brittle solids. Journal of the Mechanics and Physics of Solids 1994; 42:1397-1434.

25. Wei Y, Hutchinson JW. Models of interface separation accompanied by plastic dissipation at multiple scales. International Journal of Fracture 1999; 95:1-17.

26. Ortiz M. Microcrack coalescence and macroscopic crack growth initiation in brittle solids. International Journal of Solids and Structures 1988; 24:231-250.

27. Hillerborg A, Modéer M, Peterson PE. Analysis of crack propagation and crack growth in concrete by means of fracture mechanics and finite elements. Cement and Concrete Research 1976; 6:773-782.

28. Červenka J. Discrete crack modeling in concrete structures. Ph.D. Dissertation, University of Colorado at Boulder, 1994.

29. Carol I, Prat PC. A statically constrained microplane model for the smeared analysis of concrete cracking. In Computer Aided Analysis and Design of Concrete Structures, Bićanić N, Mang H (eds). Pineridge Press: Swansea, UK, 1990; 919-930.

30. Olofsson Th, Klisinski M, Nedar P. Inner softening bands: A new approach to localization in finite elements. In Computational Modelling of Concrete Structures, Mang H, Bićanić N, de Borst R (eds). Pineridge Press: Swansea, UK, 1994; 373-382.

31. Armero F, Garikipati K. Recent advances in the analysis and numerical simulation of strain localization in inelastic solids. In Computational Plasticity: Fundamentals and Applications, Proceedings of COMPLAS IV Owen DRJ, Oñate E (eds.) International Center for Numerical Methods in Engineering: Barcelona, 1995; 547-561.

32. Larsson R, Runesson K. Element-embedded localization band based on regularized displacement discontinuity. Journal of Engineering Mechanics ASCE 1996; 122:402-411.

33. Ohlsson U, Olofsson Th. Mixed-mode fracture and anchor bolts in concrete: analysis with inner softening bands. Journal of Engineering Mechanics, ASCE 1997; 123:1027-1033.

34. Armero F. Localized anisotropic damage of brittle materials. In Computational Plasticity: Fundamentals and Applications, Proceedings of COMPLAS V, Owen DRJ, Onate E, Hinton E (eds). International Center for Numerical Methods in Engineering: Barcelona, 1997; 635-640.

35. Chaboche JL, Girard R, Schaff A. Numerical analysis of composite systems by using interphase/interface models. Computational Mechanics 1997; 20:3-11.

36. Chaboche JL, Girard R, Levasseur P. On the interface debonding models. International Journal of Damage Mechanics 1997; 6:220-257.

37. Jirásek M. Finite elements with embedded cracks. LSC Internal Report 98/01, Swiss Federal Institute of Technology at Lausanne, 1998. 